\title{
Estudos Psicométricos da Versão Brasileira da Interpersonal Mindfulness in Parenting Scale (IMP)
}

\author{
Lauren Frantz, Veronez' \\ Felipe Valentini ${ }^{2}$ \\ Caroline Tozzi Reppold ${ }^{1}$ \\ Janaina Thais Barbosa Pacheco ${ }^{1}$ \\ ${ }^{1}$ Universidade Federal de Ciências da Saúde de Porto Alegre, Porto Alegre, Rio Grande do Sul, Brasil \\ ${ }^{2}$ Universidade São Francisco, Campinas, São Paulo, Brasil
}

\begin{abstract}
Resumo
A Interpersonal Mindfulness in Parenting Scale (IMP) é uma escala de autorrelato que avalia a utilização de habilidades de mindfulness na relação entre pais e filhos. O objetivo deste estudo foi adaptar a IMP para o contexto brasileiro e investigar suas propriedades psicométricas. A adaptação para o português brasileiro foi realizada por psicólogas com características do público-alvo do instrumento. Na etapa de busca por evidências de validade, a amostra foi composta por 221 mães de crianças entre seis e 12 anos. Utilizou-se um questionário de caracterização da amostra, IMP e Questionário de Cinco Facetas de Mindfulness (FFMQ-BR). Os resultados indicaram que os itens apresentaram composição fatorial adequada, e a escala demonstrou consistência interna adequada e se correlacionou de forma positiva com escores gerais de mindfulness (FFMQ-BR). Recomenda-se o uso da IMP em futuras investigações brasileiras que tenham como objetivo a mensuração do nível de mindful parenting em mães.

Palavras-chave: relações pais-criança, mindfulness, psicometria
\end{abstract}

Psychometric Studies of The Brazilian Version of the Interpersonal Mindfulness In Parenting Scale (IMP)

\begin{abstract}
The Interpersonal Mindfulness in Parenting Scale (IMP) is a self-report scale that assesses the use of mindfulness skills in the relationship between parents and children. This study aimed to adapt the IMP to the Brazilian context and to investigate its psychometric properties. The adaptation to Brazilian Portuguese was conducted by psychologists using characteristics of the instrument's target audience. In the search for evidence of validity, the sample consisted of 221 mothers of children between six and twelve years old. A sample characterization questionnaire, IMP, and Five Facets of Mindfulness Questionnaire (FFMQ$\mathrm{BR}$ ) were used. The results indicated that the items showed adequate factor composition, and the scale demonstrated adequate internal consistency and was positively correlated with overall mindfulness scores (FFMQ-BR). We recommend the use of IMP in future Brazilian investigations aimed at measuring the level of mindful parenting in mothers.

Keywords: parent-child relationships, mindfulness, psychometry
\end{abstract}

\section{Estudios Psicométricos de la Versión Brasileña de Interpersonal Mindfulness In Parenting Scale (IMP)}

\section{Resumen}

La Interpersonal Mindfulness in Parenting Scale (IMP) es una escala de autoinforme que evalúa el uso de las habilidades de mindfulness en la relación entre padres e hijos. El objetivo de este estudio fue adaptar la IMP al contexto brasileño e investigar sus propiedades psicométricas. La adaptación al portugués brasileño fue realizada por psicólogos con características del público objetivo del instrumento. En la búsqueda de evidencias de validez, la muestra estuvo conformada por 221 madres de niños entre seis y doce años. Se utilizó un cuestionario de caracterización de la muestra, la IMP y el Five Facets of Mindfulness Questionnaire (FFMQ-BR). Los resultados indicaron que los ítems tenían una composición factorial adecuada y la escala demostró una consistencia interna adecuada y se correlacionó positivamente con los escores generales de atención plena (FFMQ-BR). Se recomienda el uso de la IMP en futuras investigaciones brasileñas direccionadas a la medición de mindful parenting en las madres.

Palabras clave: relaciones padres-hijos, mindfulness, psicometría

\section{Introdução}

A habilidade de prestar atenção nos filhos e na própria parentalidade de forma intencional, não julgadora e centrada naquilo que acontece aqui e agora é definida como mindful parenting (Kabat-Zinn \& Kabat-Zinn, 1997). Essa abordagem implica um conjunto de práticas parentais que visam a melhorar a consciência do momento presente, a compaixão e a aceitação de si e da criança nas suas interações (Duncan, Coatsworth, \& Greenberg, 2009). Os autores apontam que adotar essa abordagem na relação com os filhos permite aos pais sair do piloto automático, diminuir a reatividade, melhorar a autorregulação e a responsividade às necessidades infantis e os ajuda a agir de acordo com seus valores e objetivos parentais (Duncan et al., 2009), 
facilitando a utilização de estratégias educativas mais efetivas e reduzindo o risco de problemas no desenvolvimento na criança (Dumas, 2005).

Ainda que o conceito de mindful parenting tenha sido mencionado há duas décadas (Kabat-Zinn \& Kabat-Zinn, 1997), os estudos nessa área iniciaram recentemente, buscando investigar os efeitos do mindful parenting no funcionamento psicológico dos pais e das crianças, assim como na qualidade da relação parental, tanto em populações clínicas, como não clínicas. Os benefícios propostos na teoria têm sido confirmados pelas pesquisas, tanto naquelas que utilizaram delineamentos transversais, como nas que propuseram intervenções.

Níveis mais altos de mindful parenting aparecem positivamente associados à maior qualidade da relação e interações mais positivas entre pais e filhos (Coatsworth, Duncan, Greenberg, \& Nix, 2010; Coatsworth et al., 2015; Duncan, Coatsworth, Gayles, Geier, \& Greenberg, 2015; Lippold et al., 2015), à satisfação parental (Singh et al., 2007), aos estilos parentais e à práticas parentais positivas (de Bruin et al., 2014; Gouveia, Carona, Canavarro, \& Moreira, 2016; Parent et al., 2016; Williams \& Wahler, 2010), à autocompaixão e ao crescimento pessoal (Benn, Akiva, Arel, \& Roeser, 2012), à capacidade de interagir com a criança de uma forma emocionalmente sintonizada e compassiva (Coatsworth, 2018) e a um melhor funcionamento psicossocial de crianças e adolescentes (Singh et al., 2007; Geurtzen et al., 2015; Parent et al., 2016). O estudo longitudinal de McKee et al. (2018) demonstra também que escores elevados de mindful parenting relacionam positivamente às boas estratégias de socialização emocional por parte dos pais, como o encorajamento à expressão de emoções.

Já baixos níveis de mindful parenting estão associados a níveis mais elevados de sintomas psicopatológicos dos pais, como estresse, ansiedade e depressão (Benn et al., 2012; Bogels \& Restifo, 2014; Corthorn \& Milicic, 2016; Gouveia et al., 2016; Moreira, Fonseca, Caiado, \& Canavarro, 2019), a mais problemas psicológicos e comportamentais nos filhos (Bögels et al., 2014; Coatsworth et al., 2010; Meppelink et al., 2016; Singh et al., 2010) e às reações parentais punitivas e geradoras de estresse para ambos (McKee et al., 2018). Além disto, associa-se com disponibilidade emocional inferior na díade mães-filhos (Benton, Coatsworth, \& Biringen, 2019) e com altos escores de comer emocional infantil e uso de comida como recompensa (Gouveia \& Moreira, 2019).
Especificamente, mindfulness pode ser entendido como a capacidade de prestar atenção intencionalmente no momento presente, com uma atitude aberta e sem julgamento (Kabat-Zinn, 1990). A partir da teoria e dos estudos empíricos sobre mindfulness, Duncan, Coatsworth e Greenberg (2009) buscaram estender esse conceito para a parentalidade, propondo um modelo teórico que explicasse como essa abordagem poderia impactar a relação entre pais e filhos e o funcionamento psicológico. Nesse modelo, cinco qualidades parentais são necessárias para desenvolver mindful parenting: (1) escutar a criança com atenção plena, que se refere a estar totalmente presente e consciente nas interações parentais enquanto ocorrem; (2) ter uma postura não julgadora e de aceitação de si mesmo e da criança, que se refere a tomar consciência das expectativas (inconscientes) a respeito de como as coisas deveriam ser e, a partir disso, aprender a aceitar traços difíceis do filho e dificuldades do papel parental existentes na vida real; (3) desenvolver consciência emocional de si e da criança, que se refere à capacidade de notar e identificar suas emoções e auxiliar o filho a entender os próprios sentimentos; (4) exercer a autorregulação na relação parental, ou seja, ser capaz de comportar-se de forma menos reativa aos comportamentos dos filhos e mais congruente com seus valores; e (5) ser compassivo consigo mesmo e com a criança, capaz de acolher a si mesmo e ao filho e ser responsivo às suas necessidades (Duncan et al., 2009).

A partir desse modelo, e com o intuito de mensurar o nível de mindful parenting em pais, foi desenvolvida a Interpersonal Mindfulness in Parenting Scale (IMP), uma escala de autorrelato para acessar essas cinco dimensões (Duncan, 2007). A primeira versão da escala continha apenas dez itens e foi construída a partir de instrumentos já existentes de mindfulness, como o Kentucky Inventory of Mindfulness Skills (Baer, Smith, \& Allen, 2004), o Mindful Attention and Awareness Scale (Brown \& Ryan, 2003) e a subescala de mindfulness do Self-Compassion Scale (Neff, 2003). O estudo inicial de validação, desenvolvido nos Estados Unidos, indicou que empiricamente o instrumento agregava quatro fatores: (1) atenção ao momento presente, (2) consciência emocional do momento presente, (3) não reatividade e (4) não julgamento e aceitação. A consistência interna, medida pelo alfa de Cronbach, foi de 0,72 para o escore total. Evidências de validade convergente e discriminante da escala foram demonstradas em relação a outros construtos parentais e a mindfulness (Duncan et al., 2007). A versão da IMP de 
dez itens foi utilizada no estudo piloto de uma intervenção para mães e crianças entre 10 e 14 anos de idade (Coatsworth et al., 2010) e os resultados indicaram que o construto mindfulparenting mediou os efeitos da intervenção em resultados-chave relacionados ao funcionamento materno e infantil, como a qualidade da relação entre mães e filhos e as estratégias disciplinares utilizadas.

Com o objetivo de avaliar mais detalhadamente as cinco dimensões propostas pelo modelo teórico, Duncan et al. (2009) expandiram a escala para uma versão de 31 itens, com pelo menos cinco itens para avaliar cada fator hipotetizado inicialmente. Entretanto, os autores não realizaram estudos para avaliar as propriedades psicométricas da última versão da IMP. Contudo, autorizaram seu uso na Holanda, e de Bruin et al. (2014) foram pioneiros na busca de evidências de validade do instrumento nesse país. Em seu primeiro estudo, conduziram uma análise fatorial exploratória para avaliar dados coletados em uma amostra de 866 mães, que resultou na extração de seis fatores, diferindo da hipótese dos autores originais.

Em um segundo estudo, com uma amostra diferente, composta por 199 mães, a estrutura de seis fatores foi corroborada por meio de uma análise fatorial confirmatória. As dimensões finais do modelo holandês foram: escutar com atenção plena, compaixão pela criança, não julgamento e aceitação da função parental, não reatividade emocional na parentalidade, consciência emocional da criança e consciência emocional de si mesmo. Dois itens ( 3 e 6 ) foram removidos da escala por apresentarem fracas propriedades psicométricas. Portanto, a versão final da IMP na Holanda ficou composta por 29 itens, tendo apresentado alfa de Cronbach de 0,89 no primeiro estudo e 0,85 , no segundo. Além dos dados apresentados, evidências de validade foram obtidas por meio de correlações significativas entre as subescalas da IMP e medidas de otimismo, depressão, qualidade de vida, estilos parentais e mindfulness (de Bruin et al., 2014).

Em Portugal, Moreira e Canavarro (2017) também buscaram investigar a estrutura fatorial da IMP, assim como sua confiabilidade e suas evidências de validade convergente e de estrutura interna. As análises exploratórias apontaram inicialmente uma solução de seis fatores, assim como na versão holandesa. Porém, a consistência interna de um deles (consciência emocional de si mesmo) foi muito baixa (alfa de Cronbach $=$ $0,42)$, considerada inaceitável. Cite-se que tal fator era composto exclusivamente por dois itens (itens 3 e 6 ), que apresentaram carga fatorial $<0,30$, o que levou os autores a optarem pela exclusão desse fator.

Assim, a versão final da escala foi composta por 29 itens e cinco dimensões e apresentou alfa de Cronbach total igual a 0,89 . Além disso, foram encontradas correlações significativas entre as subescalas da IMP e instrumentos que avaliam autocompaixão (Self-Compassion Scale - Short Form) e estresse parental (Parenting Stress Scale), evidenciando validade convergente do instrumento. Em um segundo momento, foi realizada também uma análise fatorial confirmatória, com os dados de uma nova amostra, composta por 323 mães, que testou o modelo de cinco fatores. Os resultados indicaram a adequação desse modelo $[\chi 2(365)=624,16$; $p<0,001 ; \chi 2 / d f=1,71 ; \mathrm{CFI}=0,93$; RMSEA $=0,05$; $\mathrm{SRMR}=0,05]$. As análises de consistência interna apresentaram resultados satisfatórios, com alfa de Cronbach variando entre 0,70 e 0,86 para as subescalas e 0,93 para o escore total.

Assim, dada a pertinência dos estudos desenvolvidos sobre mindful parenting sobre a qualidade da relação entre mães e filhos e seus desdobramentos sobre a saúde emocional e o desenvolvimento humano e considerando que, no Brasil, não há instrumentos validados para avaliar tal construto, a presente pesquisa visa a preencher essa lacuna. Para tanto, o objetivo deste estudo foi adaptar a IMP para a cultura brasileira e investigar suas propriedades psicométricas por meio da estimativa de sua precisão e da busca de evidências de validade baseada na estrutura interna (estrutura fatorial) e em variáveis externas (validade convergente).

\section{Método}

\section{Participantes}

Para a etapa do estudo de adaptação da escala, participaram duas experts em Psicologia que tinham domínio da língua portuguesa de Portugal e da língua portuguesa do Brasil (uma portuguesa residente no Brasil e a outra brasileira residente em Portugal), com o objetivo de adaptar a linguagem utilizada na IMP para o português brasileiro. Em uma segunda etapa, cujo objetivo foi avaliar a síntese do instrumento após a adaptação da linguagem, participaram três mães, doutoras em Psicologia, que tinham filhos entre seis e doze anos de idade e residiam com eles. A escolha das participantes com esse perfil buscou atender às sugestões de Borsa, Damásio e Bandeira (2012) para a realização das etapas de avaliação da síntese por experts e avaliação 
pelo público-alvo, realizando, então, essas duas etapas conjuntamente.

Para a etapa do estudo de evidências de validade, a amostra foi composta por 221 mães de crianças em idade escolar. Para a delimitação da idade das crianças foi considerada uma faixa desenvolvimental com demandas semelhantes sobre as mães, buscando-se evitar crianças pequenas ou em idade pré-escolar e adolescentes. Observou-se, ainda, que os estudos que utilizaram a IMP não são homogêneos com relação à idade dos filhos (de Bruin et al., 2014; Moreira \& Canavarro, 2017; Moreira et al., 2019). Os critérios de inclusão amostral foram: ter pelo menos um filho com idade entre seis e doze anos, ser maior de 18 anos e residir com o(s) filho(s). A idade média das participantes foi de 38,6 anos $(D P=6,1)$ para as mães e de 8,3 anos $(D P=2)$ para os filhos.

A maioria da amostra foi composta de mulheres casadas/morando com companheiro (80,5\%), graduadas ou pós-graduadas $(84,2 \%)$, residentes em capitais ou regiões metropolitanas brasileiras $(50,7 \%)$, que trabalham pelo menos meio turno $(77,3 \%)$. No total, $45,7 \%$ possuíam renda familiar mensal maior que $\mathrm{R} \$$ 6.000. Em relação ao número de filhos, $45,7 \%$ da amostra possuía dois filhos e 44,3\% tinham filho único. Quanto ao sexo, 54,3\% das crianças eram meninas e $68,3 \%$ estudavam em escolas privadas. Os instrumentos foram respondidos somente pelas mães.

\section{Instrumentos}

Para a coleta de dados foram utilizados os seguintes instrumentos:

- Questionário de Caracterização da Amostra. Instrumento criado pelas autoras para este estudo, composto de 17 itens e utilizado para mapear dados sociodemográficos.

- Interpersonal Mindfulness in Parenting Scale (IM-P) (Moreira \& Canavarro, 2017). Instrumento composto por 31 itens e respondido por meio de uma escala Likert de cinco pontos, variando entre 1 (nunca) a 5 (sempre). A versão utilizada avalia cinco dimensões do mindful parenting: escutar o filho com atenção plena, aceitação não julgadora de si e da criança, consciência emocional da criança, autorregulação na relação parental e compaixão pela criança. Os resultados da escala derivam um escore total e um escore por fator, sendo que alta pontuação indica nível mais elevado de mindful parenting.
O instrumento pode ser respondido por pais ou mães, não havendo versões para as crianças. Neste estudo, foi solicitado para mães com mais de um filho que escolhessem apenas uma criança sobre a qual responderiam ao instrumento.

- Questionário de Cinco Facetas de Mindfulness (FFMQ-BR) (Barros, Kozasa, Souza, \& Ronzani, 2014). Instrumento de 39 itens respondido por meio de uma escala Likert de cinco pontos. Sua estrutura fatorial original apresenta cinco facetas: Observar, que se refere a notar e prestar atenção a estímulos internos e externos, como sensações, emoções, cognições, cheiros, sons; Descrever, que se refere à capacidade de nomear as experiências internas observadas; Agir com consciência, que envolve prestar atenção às atividades do momento presente, em contraste à ideia de piloto automático; Não julgamento das experiências internas, que implica adotar uma postura não avaliativa dos pensamentos e emoções; e Não reagir às experiências internas, que se refere a permitir que pensamentos e sentimentos apareçam e desapareçam, sem fixar-se ou ser influenciado e conduzido por eles. $\mathrm{Na}$ versão brasileira, após estudo empírico, a faceta "descrição" foi dividida em positiva e negativa, e a faceta "ação com consciência" foi dividida em agir no piloto automático e agir distraidamente. No presente estudo, o alfa de Cronbach foi de 0,88 para o total do instrumento. Os resultados por faceta foram não julgamento $(\alpha=0,81)$, ação com consciência - piloto automático $(\alpha=0,87)$, observação $(\alpha=0,70)$, descrição - itens positivos $(\alpha=0,82)$, descrição - itens negativos $(\alpha=0,74)$, não reatividade $(\alpha=0,72)$ e ação com consciência - distração $(\alpha=0,77)$.

\section{Procedimentos de Coleta de Dados e Aspectos Éticos}

Inicialmente, foi solicitada autorização das autoras da versão portuguesa da escala (Moreira \& Canavarro, 2017) para a adaptação dela. Após autorizado, duas experts em Psicologia (uma delas brasileira residente em Portugal e a outra portuguesa residente no Brasil) realizaram a tradução da IMP do português de Portugal para o português brasileiro. Em um segundo momento, as autoras deste estudo realizaram uma síntese entre as versões recebidas, criando uma terceira versão do instrumento. A versão sintetizada foi avaliada por três mães, doutoras em Psicologia, sem familiaridade com o 
construto que estava sendo medido, a fim de que fosse fornecido feedback sobre layout, adequação e clareza dos itens e do rapport, constituindo-se as etapas de avaliação da síntese por experts e avaliação pelo público-alvo (Borsa, Damásio, \& Bandeira, 2012). Concluída essa etapa, foram realizadas as modificações pertinentes e elaborada a versão final do instrumento, aplicada na amostra do estudo que investigou as propriedades psicométricas do instrumento adaptado.

A amostra do estudo de busca de evidências de validade foi recrutada por conveniência na população geral por intermédio da divulgação da pesquisa em mídias sociais digitais (Facebook e WhatsApp), pela técnica snowball (bola de neve). Esta é uma técnica não probabilística, em que os indivíduos participantes da fase inicial da pesquisa, aos quais o pesquisador tem acesso, convidam novos participantes por meio da sua própria rede (Nunes \& Dewes, 2013).

A aplicação dos instrumentos ocorreu por meio de formulários disponibilizados na Internet às participantes por meio de um link do SurveyMonkey, uma plataforma de questionários on-line. Os dados foram coletados entre dezembro de 2017 e março de 2018. O cálculo amostral foi feito com base nas orientações de Pasquali (2003), que indica o número mínimo de cinco respondentes por item do instrumento, uma vez que a estrutura fatorial da escala utilizada já era conhecida, o que apontava uma previsão de tamanho amostral de, pelo menos, 155 participantes. Durante o período em que a plataforma de coleta de dados esteve aberta, 323 pessoas aceitaram participar do estudo, porém, nem todas responderam por completo ao questionário sociodemográfico e ao IMP, sendo excluídas do estudo. Assim, o $n$ considerado para esta pesquisa foi de 221 mulheres.

Este projeto está de acordo com a Resolução $n^{\circ}$ 466 de 12 de dezembro de 2012, que trata das diretrizes e normas regulamentadoras de pesquisas envolvendo seres humanos, e foi aprovado pelo Comitê de Ética em Pesquisa (373: 2.137.922), por meio do parecer de n. 2.137.922. Antes de iniciar a coleta de dados, o Termo de Consentimento Livre e Esclarecido (TCLE) foi apresentado a todas as participantes na primeira tela da plataforma, com o objetivo de esclarecer os objetivos da pesquisa e convidá-las para participar do estudo.

\section{Procedimentos de Análise de Dados}

Para avaliar a estrutura do instrumento, foi adotado o procedimento de análise fatorial confirmatória (AFC), utilizando-se a modelagem por equações estruturais por meio de modelo restrito, no qual crossloadings são fixados em zero a priori. Foi testada uma estrutura com cinco fatores, conforme o modelo original da escala. Para o ajuste do modelo, foram avaliados os indicadores qui-quadrado $(\chi 2)$, CFI, TLI e RMSEA. Os parâmetros do modelo foram estimados por meio de Weighted Least Squares Mean and Variance Adjusted (WLSMV), e os itens foram configurados como categóricos ordinais. As análises foram realizadas por meio do software Mplus (v. 8.0). A precisão dos escores foi estimada pelo alfa de Cronbach.

\section{Resultados}

Para a tradução da IMP, foi solicitado às tradutoras que evitassem o uso de termos ou expressões regionais, a fim de que o instrumento pudesse ser compreendido e aplicado em diferentes lugares do país. Alguns itens foram traduzidos igualmente pelas profissionais, enquanto outros diferiram nas duas versões. Por exemplo, no item 2, foi sugerido por uma delas "Quando estou aborrecido com o meu filho, consigo identificar como me sinto antes de agir" e por outra "Quando estou aborrecido com o meu filho, consigo perceber como estou me sentindo antes de agir"; no item 5, enquanto uma das avaliadoras o traduziu como "Frequentemente, reajo rápido demais ao que o meu filho diz ou faz" outra sugeriu "Frequentemente, reajo muito depressa ao que o meu filho diz ou faz".

A partir dessas duas versões, foi realizado o processo de síntese por duas autoras do estudo, comparando as discrepâncias e diferenças entre elas e tomando como parâmetro de avaliação o instrumento original. Após essa etapa, a versão sintetizada foi aplicada em três doutoras em Psicologia, sem familiaridade com o construto de mindfulparenting e que tivessem pelo menos um filho com idade entre 6 e 12 anos. Após responder ao questionário, cada uma delas reportou sua impressão geral da escala, assim como feedbacks a respeito do layout, rapport, formulação dos itens e demais sugestões. Uma das entrevistadas apontou que o instrumento a fez refletir sobre as próprias atitudes em relação à filha, o que é um dado qualitativo importante e esperado que ocorra no preenchimento da escala. Outra apontou a importância de que as frases estivessem também no feminino, acrescentando sempre um "(a)" nos itens, como, por exemplo, no item 9 (Faço com pressa atividades com o meu filho(a), sem estar realmente atento(a) a ele(a). As três entrevistadas não relataram dificuldades no preenchimento da escala, descreveram o layout 
e o rapport como apropriados e o instrumento como de grande valia para a área estudada.

Com o intuito de investigar a composição fatorial da escala, testou-se um modelo com os cinco fatores. $\mathrm{Na}$ primeira análise, foram excluídos os itens 3 e 6 que, apresentaram carga fatorial abaixo do recomendado (menor que 0,30 ) e juntos compunham um fator de dois itens com alfa de Cronbach igual a 0,42 (Moreira \& Canavarro, 2017). Os dados obtidos evidenciaram que o modelo testado de cinco fatores obteve índices de ajuste limítrofes: $\chi^{2}(367)=768,28, p<0,001 ; \chi^{2} / d f$ $=1,98 ; \mathrm{CFI}=0,89 ; \mathrm{TLI}=0,88 ; \mathrm{RMSEA}=0,07$.

Conforme observa-se na Tabela 1 , à exceção do item 5 , todas os demais itens apresentaram cargas fatoriais padronizadas significativas $(\phi<0,001)$, variando de 0,32 (item 10) a 0,87 (item 31). Ressalta-se que o item 5 foi excluído da versão final e que os demais itens foram retidos nos mesmos fatores que eram

Tabela 1.

Cargas Fatoriais dos Itens Retidos na Análise Fatorial Confirmatória

\begin{tabular}{|c|c|c|c|c|c|}
\hline Item & $\begin{array}{c}\text { Fator } 1 \\
\text { NJA }\end{array}$ & $\begin{array}{c}\text { Fator } 2 \\
\text { ARP }\end{array}$ & $\begin{array}{c}\text { Fator } 3 \\
\text { CPC }\end{array}$ & $\begin{array}{c}\text { Fator } 4 \\
\text { EAP }\end{array}$ & $\begin{array}{c}\text { Fator } 5 \\
\text { CEC }\end{array}$ \\
\hline 23 & 0,79 & & & & \\
\hline 18 & 0,57 & & & & \\
\hline 26 & 0,56 & & & & \\
\hline 17 & 0,47 & & & & \\
\hline 20 & 0,42 & & & & \\
\hline 15 & 0,37 & & & & \\
\hline 10 & 0,32 & & & & \\
\hline 14 & & 0,70 & & & \\
\hline 16 & & 0,68 & & & \\
\hline 11 & & 0,67 & & & \\
\hline 29 & & 0,61 & & & \\
\hline 08 & & 0,59 & & & \\
\hline 21 & & 0,58 & & & \\
\hline 02 & & 0,48 & & & \\
\hline 31 & & & 0,87 & & \\
\hline 27 & & & 0,79 & & \\
\hline 25 & & & 0,71 & & \\
\hline 28 & & & 0,70 & & \\
\hline 04 & & & 0,68 & & \\
\hline 07 & & & 0,64 & & \\
\hline 24 & & & & 0,83 & \\
\hline 19 & & & & 0,80 & \\
\hline 09 & & & & 0,64 & \\
\hline 13 & & & & 0,63 & \\
\hline 01 & & & & 0,56 & \\
\hline 12 & & & & & 0,72 \\
\hline 30 & & & & & 0,72 \\
\hline 22 & & & & & 0,70 \\
\hline
\end{tabular}

Nota. Subescalas do IMP: NJA = Não Julgamento e Aceitação na parentalidade; ARP = Autorregulação na parentalidade; CPC = Compaixão pela Criança; EAP = Escutar com Atenção Plena; CEC = Consciência Emocional da Criança. 
alocados no modelo proposto por Moreira e Canavarro (2017), sendo a magnitude das cargas fatoriais obtidas, em sua maioria, semelhantes às apresentadas por esses autores.

A consistência interna da escala, calculada por meio do coeficiente alfa de Cronbach, foi igual a 0,89. Respectivamente, os alfas de Cronbach dos fatores isolados foram: Não julgamento e Aceitação na parentalidade = 0,70; Autorregulação na parentalidade $=0,79$; Compaixão pela criança $=0,80$; Escutar com atenção plena $=$ 0,82 e Consciência emocional da criança $=0,70$.

As evidências de validade convergente do instrumento podem ser observadas na Tabela 2. Correlações significativas foram encontradas entre os escores totais e entre as subescalas do IMP e do FFMQ, utilizado para avaliar o nível de mindfulness.

\section{Discussão}

A tradução e a adaptação transcultural são os primeiros passos em direção à validação de um instrumento psicológico (Borsa et al., 2012). Nesta investigação, foi realizada a tradução da versão portuguesa para o português brasileiro por duas tradutoras qualificadas para tal função: profissionais de Psicologia e com vivências nos dois países. Além disso, a escala foi avaliada por representantes do público-alvo antes de chegar à versão final, utilizada nesta pesquisa. $\mathrm{Na}$ sua versão brasileira, chama-se Escala de Mindfulness na Parentalidade.

Os dados mostraram que a escala obteve boas propriedades psicométricas. A consistência interna do instrumento se mostrou adequada para todos os fatores do instrumento e para o escore total na versão brasileira. No que se refere à estrutura interna da IMP, os dados obtidos na análise fatorial confirmatória evidenciaram que o modelo de cinco fatores utilizado na versão portuguesa (Moreira \& Canavarro, 2017) apresentou bons índices de ajuste, permitindo a sua replicação para a população brasileira. Apenas o item 5 ("Frequentemente, reajo rápido demais ao que o meu filho(a) diz ou faz"), que originalmente pertencia ao fator Autorregulação na parentalidade apresentou baixa carga fatorial (igual a 0,23$)$ e, por esse motivo, foi excluído do instrumento. Essa subescala é bastante similar ao modelo original (Duncan et al., 2009) e avalia a habilidade dos pais em regular suas próprias emoções e comportamentos na relação com seus filhos.

O processo de adaptação e busca de evidências de validade no Brasil resultou em uma escala de 28 itens, composta por cinco subescalas. Não julgamento e

Tabela 2.

Resultados das Correlações de Spearman obtidos entre o IMP (fatores e total) e o FFMQ (fatores e total)

\begin{tabular}{lccccccccc}
\hline & & NJEI & ACPA & OBS & DPOS & DNEG & NREA & ACD & FFMQ Total \\
\hline NJA & $r$ & 0,47 & 0,49 & 0,17 & 0,20 & 0,28 & 0,25 & 0,39 & 0,55 \\
& $p$ & 0,00 & 0,00 & 0,02 & 0,00 & 0,00 & 0,00 & 0,00 & 0,00 \\
ARP & $r$ & 0,30 & 0,33 & 0,21 & 0,29 & 0,37 & 0,32 & 0,20 & 0,49 \\
& $p$ & 0,00 & 0,00 & 0,00 & 0,00 & 0,00 & 0,00 & 0,00 & 0,00 \\
CPC & $r$ & 0,21 & 0,24 & 0,23 & 0,24 & 0,24 & 0,26 & 0,21 & 0,39 \\
& $p$ & 0,00 & 0,00 & 0,00 & 0,00 & 0,00 & 0,00 & 0,00 & 0,00 \\
EAP & $r$ & 0,28 & 0,48 & 0,16 & 0,09 & 0,20 & 0,19 & 0,43 & 0,40 \\
& $p$ & 0,00 & 0,00 & 0,03 & 0,23 & 0,00 & 0,01 & 0,00 & 0,00 \\
CEC & $r$ & 0,04 & 0,34 & 0,07 & 0,18 & 0,20 & 0,13 & 0,31 & 0,27 \\
& $p$ & 0,63 & 0,00 & 0,34 & 0,01 & 0,00 & 0,07 & 0,00 & 0,00 \\
\multirow{2}{*}{ Total IMP } & $r$ & 0,41 & 0,53 & 0,23 & 0,28 & 0,37 & 0,32 & 0,42 & 0,61 \\
& $p$ & 0,00 & 0,00 & 0,00 & 0,00 & 0,00 & 0,00 & 0,00 & 0,00 \\
\hline
\end{tabular}

Nota. Subescalas do IMP: NJA = Não Julgamento e Aceitação na parentalidade; ARP = Autorregulação na parentalidade; CPC $=$ Compaixão pela Criança; EAP = Escutar com Atenção Plena; CEC = Consciência Emocional da Criança; Subescalas no FFMQ: NJEI = Não Julgar Experiência Interna; ACPA = Agir com Consciência: Piloto Automático; OBS = Observar; DPOS = Descrever Positivo; DNEG = Descrever Negativo; NREA = Não Reatividade; ACD = Agir com Consciência: Distração. 
Aceitação na parentalidade foi composta por sete itens (10, 15, 17, 18, 20, 23 e 26), assim como Autorregulação na parentalidade $(2,8,11,14,16,21$ e 29$)$; a subescala Compaixão pela criança agrupou seis itens $(4,7,25,27$, 28 e 31); Escutar com atenção plena foi composta por cinco itens $(1,9,13,19$ e 24$)$ e a subescala Consciência emocional da criança reuniu três itens $(12,22,30)$.

A validade convergente foi evidenciada pelas correlações entre mindfulness e mindfulparenting. Ao observar as subescalas que se correlacionaram com mais força, é possível levantar hipóteses sobre essa interação. $\mathrm{Na}$ IMP, tanto Escutar com atenção plena como Consciência emocional da criança correlacionaram-se com as duas facetas de ação com consciência do FFMQ, que contrastam com a ideia de piloto automático e de distração. Estar presente e consciente ao que se faz no momento, como, por exemplo brincar com o filho, é necessário para que se escute e perceba atentamente as necessidades da criança. Além disso, quando os pais são sensíveis tanto ao conteúdo do que o filho diz como ao seu tom de voz, expressões faciais e linguagem corporal, podem usar essas pistas para detectar suas necessidades e agir de acordo com elas (Duncan et al., 2009). Ao perceber com maior acurácia os sentimentos e pensamentos dos filhos, os pais conseguem aumentar sua empatia e escolher de forma mais consciente como irão responder às situações, ao invés de apenas reagir de forma automática, o que também reduz conflitos e desentendimentos (Morris, Silk, Steinberg, Myers, \& Robinson, 2007).

A Autorregulação na parentalidade (IMP) apresentou correlação mais elevada, embora fraca $(r=0,37$; $p<0,001)$, com Descrever negativo (FFMQ), que é a capacidade de nomear experiências vivenciadas (itens com formulação negativa) (Barros et al., 2014). Pode-se pensar que, ao ser capaz de colocar em palavras o que sentem, as mães tenham maior consciência do seu mundo interno e das suas reações, o que auxiliaria também a modelar os filhos na forma como lidam com suas emoções, propiciando um importante efeito socializador (Morris et al., 2007). A faceta Autorregulação também apresentou correlações significativas, mas fracas, com os fatores Não julgar experiência interna $(r$ $=0,30 ; p<0,001)$, Agir com consciência: piloto automático $(r=0,32 ; p<0,001)$, e Não-reatividade $(r=$ $0,31 ; p<0,001)$. Com isso, pode-se pensar que mães menos reativas e críticas em relação às suas dificuldades internas são também aquelas que se comportam mais de acordo com os seus valores e objetivos e menos de acordo com seus impulsos.
A faceta Não julgamento e aceitação na parentalidade do IMP apresentou correlações mais fortes com Agir com consciência: piloto automático $(r=$ $0,48 ; p<0,001)$, e Não julgar experiência interna $(r$ $=0,46 ; p<0,001)$. Ou seja, prestar atenção às suas ações no aqui e agora e possuir a capacidade de aceitar suas experiências internas sem julgamentos pode facilitar que as mães generalizem essas características para suas práticas parentais. É importante ressaltar que, quando se trata de mindfulness, aceitação não significa em nenhum momento resignação, e sim entrar em contato com aquilo que ocorre no momento presente sem julgar (Kabat-Zinn, 1990). Na parentalidade, é aceitar que é extremamente desafiador criar um filho e que cometer erros é parte deste processo (Duncan et al., 2009).

Já Compaixão pela criança não obteve $r>0,30 \mathrm{em}$ relação a nenhuma faceta do FFMQ, ainda que o escore total tenha apresentado correlação de $r=0,395$. Provavelmente, isso ocorreu porque o instrumento FFMQ não possui nenhum fator específico que avalie compaixão/autocompaixão, embora a relação entre esses dois construtos e mindfulness já esteja bem estabelecida (Germer \& Barnhofer, 2017). O estudo de Moreira, Carona, Silva, Nunes e Canavarro (2015) evidencia que níveis mais altos de autocompaixão ajudam os pais a adotar uma abordagem mais consciente em sua relação com os filhos. Pais autocompassivos são mais gentis consigo mesmo e menos críticos em relação às suas habilidades parentais, o que está relacionado também a níveis mais altos de mindful parenting.

O papel protetivo que mindful parenting pode desempenhar na relação entre pais e filhos é observado nos resultados do estudo de Moreira et al. (2019). Os autores investigaram a relação entre conflitos família-trabalho, ansiedade, depressão e estresse parental e o tipo de trabalho dos pais. Os resultados indicaram correlações negativas acima de $0,30(p<0,001)$ entre as subescalas do IMP e sintomas ansiosos, sintomas depressivos e estresse parental.

Também utilizando o IMP, o trabalho de Gouveia, Canavarro e Moreira (2020) investigou a relação entre as dimensões de mindful parenting com sobrepeso infantil. Os resultados mostraram que mães de crianças com sobrepeso ou obesidade apresentaram níveis mais baixos de consciência emocional da criança e aceitação sem julgamentos e sugerem que habilidades de mindful parenting podem ajudar as mães a adotarem práticas mais saudáveis de alimentação infantil adaptadas ao peso da criança. 
Considerando-se esses estudos, a disponibilização do IMP para estudos nacionais vem ao encontro do aumento da produção internacional sobre minful parenting relacionado com variáveis de saúde mental e de desenvolvimento infantil. Além disso, integra os estudos que têm adaptado o IMP para outras culturas (Kim, Krägeloh, Medvedev, Duncan, \& Singh, 2019; Moreira \& Gouveia, 2017).

\section{Considerações Finais}

O principal objetivo deste estudo foi adaptar a IMP para o contexto brasileiro e investigar a estimativa de precisão e as evidências de validade, mediante análise da sua estrutura interna e estudo de validade convergente. A escala demonstrou boa consistência interna e uma correlação positiva com FFMQ. As análises confirmaram a estrutura fatorial da versão portuguesa, indicando que mindful parenting é avaliado nessa escala por meio de cinco dimensões.

Algumas limitações deste trabalho devem ser apontadas. Primeiro, o fato de todas as medidas utilizadas basearem-se em autorrelato, o que pode, eventualmente, ter enviesado os dados e introduzido efeitos de desejabilidade social. Segundo, a amostra foi composta apenas por mães; portanto, os resultados não são generalizáveis para amostras compostas por pais. Terceiro, a amostra apresentou renda e nível de escolaridade elevado, deixando em aberto mais uma vez a questão sobre a possibilidade de generalização dos dados e do uso do instrumento por mães com renda e escolaridade diversas. Assim, pesquisas futuras devem ser conduzidas para fortalecer as evidências de validade do instrumento no país, assim como para verificar se a estrutura fatorial se mantém igual em diferentes culturas e contextos.

Contudo, as forças do estudo também devem ser notadas. Este trabalho representa uma contribuição importante no campo de pesquisa do mindfulness e da parentalidade, sendo pioneiro na investigação das evidências de validade da Interpersonal Mindfulness In Parenting Scale para a população brasileira. A avaliação minuciosa das correlações entre as subescalas dos instrumentos utilizados possibilitou levantar hipóteses que auxiliem no entendimento da relação entre esses construtos. Ainda que mais estudos sejam necessários, as evidências iniciais de validade obtidas neste estudo são robustas. Desse modo, o uso dessa escala em futuras investigações brasileiras que tenham como objetivo a mensuração do nível de mindful parenting dos indivíduos é recomendável.

\section{Referências}

Baer, R., Smith, G., \& Allen, K. (2004). Assessment of mindfulness by self-report: the Kentucky Inventory of Mindfulness Skills. Assessment, 11(3), 191-206. doi:10.1177/1073191104268029

Barros, V. V., Kozasa, E. H., Souza, I. C. W., \& Ronzani, T. M. (2014). Validity evidence of the Brazilian version of the Five Facet Mindfulness. Psicologia: Teoria E Pesquisa, 30(3), 317-327. doi: 10.1590/ S0102-37722014000300009

Benn, R., Akiva, T., Arel, S., \& Roeser, R. W. (2012). Mindfulness training effects for parents and educators of children with special needs. Developmental Psychology, 48(5), 1476-1487. http://dx.doi. org/10.1037/a0027537

Benton, J., Coatsworth, D. \& Biringen, Z. (2019) Examining the Association Between Emotional Availability and Mindful Parenting. J Child Fam Stud 28, 1650-1663. https://doi.org/10.1007/ s10826-019-01384-x

Bögels, S., Hellemans, J., van Deursen, S., Römer, M., \& van der Meulen, R. (2014). Mindful parenting in mental health care: effects on parental and child psychopathology, parental stress, parenting, coparenting, and marital functioning. Mindfulness, 5(5), 536-551. doi:10.1007/s12671-013-0209-7.

Bögels, S., \& Restifo, K. (2014). Mindful parenting: a guide for mental health practitioners. New York: Springer.

Borsa, J. C, Damásio, B. F., \& Bandeira, D. R. (2012). Adaptação e validação de instrumentos psicológicos entre culturas: algumas considerações. Paidéia (Ribeirão Preto), 22(53), 423-432. https://dx.doi. org/10.1590/S0103-863X2012000300014

Brown, K. W., \& Ryan, R.M. (2003). The benefits of being present: Mindfulness and its role in psychological well-being. Journal of Personality and Social Psychology, 84(4), 822-848. doi: 10.1037/0022-3514.84.4.822

Coatsworth, J; Timpe, Z; Nix, R.; Duncan, L.; Greenberg, M. (2018). Changes in Mindful Parenting: Associations With Changes in Parenting, ParentYouth Relationship Quality, and Youth Behavior. Journal of the Society for Social Work and Research, 9(4), 511-529. doi:10.1086/701148.

Coatsworth, J. D., Duncan, L. G., Greenberg, M. T., \& Nix, R. L. (2010). Changing parent's mindfulness, 
child management skills and relationship quality with their youth: Results from a randomized pilot intervention trial. Journal of Child and Family Studies, 19(2), 203-217. doi: 10.1007/s10826-009-9304-8

Coatsworth, J. D., Duncan, L. G., Nix, R. L., Greenberg, M. T., Gayles, J. G., Bamberger, K. T., Demi, M. A. (2015). Integrating mindfulness with parent training: effects of the Mindfulness-Enhanced Strengthening Families Program. Developmental Psychology, 51(1), 26-35. doi: 10.1037/a0038212

Corthorn, C. (2018). Benefits of mindfulness for parenting in mothers of preschoolers in Chile. Frontiers in Psychology, 9, 1443-1443. doi: 10.3389/ fpsyg.2018.01443.672-1683

Corthorn, C., \& Milicic, N. (2016). Mindfulness and parenting: A correlational study of nonmeditating mothers of preschool children. Journal of Child and Family Studies, 25(1), 1.

de Bruin, E., Zijlstra, B. H., Geurtzen, N., van Zundert, R. P., van de Weijer-Bergsma, E., ... Hartman, E. (2014). Mindful parenting assessed further: Psychometric properties of the Dutch version of the Interpersonal Mindfulness in Parenting scale (IM-P). Mindfulness, 5(2), 200-212. doi:10.1007/ s12671-012-0168-4

Dumas, J. (2005). Mindfulness-based parent training: Strategies to lessen the grip of automaticity in families with disruptive children. Journal of Clinical Child and Adolescent Psychology, 34(4), 779-791. doi: 10.1207/s15374424jccp3404

Duncan, L. (2007). Assessment of mindful parenting among parents of early adolescents: Development and validation of the Interpersonal Mindfulness in Parenting Scale. Doctoral dissertation, The Pennsylvania State University, USA.

Duncan, L. G., Coatsworth, J. D., Gayles, J. G., Geier, M. H., \& Greenberg, M. T. (2015). Can mindful parenting be observed? Relations between observational ratings of mother-youth interactions and mothers' self-report mindful parenting. Journal of Family Psychology, 29(2), 276-282. doi:10.1037/ a0038857

Duncan, L. G., Coatsworth, J. D., \& Greenberg, M. T. (2009). A model of mindful parenting: implications for parent-child relationships and prevention research. Clinical Child and Family Psychology Review, 12(3), 255-270. doi: 10.1007/s10567-009-0046-3
Germer, C., \& Barnhofer, T. Mindfulness and compassion In Paul Gilbert (2017) Compassion: Concepts, Research and Applications. Taylor \& Francis, 69-85. ISBN 9781317189480.

Geurtzen, N., Scholte, R. J., Engels, R. M. E., Tak, Y., \& van Zundert, R. P. (2015). Association between mindful parenting and adolescents' internalizing problems: non-judgmental acceptance of parenting as core element. Journal of Child and Family Studies, 24(4), 1117-1128. doi:10.1007/s10826-014-9920-9

Gouveia, M. J., Canavarro, M. C., \& Moreira, H. (2020). The role of mindful parenting and children's weight in mothers' child-feeding practices. Eating and Weight Disorders-Studies on Anorexia, Bulimia and Obesity, 25(2), 427-435. doi: 10.1007/ s40519-018-0615-x

Gouveia, M. J., Carona, C., Canavarro, M. C., \& Moreira, H. (2016). Self- compassion and dispositional mindfulness are associated with parenting styles and parenting stress: The mediating role of mindful parenting. Mindfulness, 1(13), doi:10.1007/ s12671-016-0507-y

Gouveia, M. J. \& Moreira, H. (2019). How can mindful parenting be related to emotional eating and overeating in childhood and adolescence? The mediating role of parenting stress and parental child-feeding practices. Appetite, vol. 138, 102114, doi: 10.1016/j.appet.2019.03.021

Kabat-Zinn, J. (1990). Full catastrophe living: Using the wisdom of your body and mind to face stress, pain, and illness. New York: Dell Publishing.

Kabat-Zinn, M., \& Kabat-Zin, J. (1997). Everyday blessings: The inner work of mindful parenting. New York: Hyperion.

Kim, E., Krägeloh, C. U., Medvedev, O. N., Duncan, L. G., \& Singh, N. N. (2019). Interpersonal mindfulness in parenting scale: testing the psychometric properties of a korean version. Mindfulness, 10(3), 516-528. doi: 10.1007/s12671-018-0993-1

Lippold, M., Duncan, L., Coatsworth, J. D., Nix, R., \& Greenberg, M. (2015). Understanding how mindful parenting may be linked to mother-adolescent communication. Journal of Youth and Adolescence, 44(9), 1663-1673. doi:10.1007/s10964-015-0325-x

McKee, L. G., Parent, J., Zachary, C. R., \& Forehand, R. (2018). Mindful parenting and emotion 
socialization practices: Concurrent and longitudinal associations. Fam. Proc., 57, 752-766

Meppelink, R., de Bruin, E. I., Wanders-Mulder, F. H., Vennik, C. J., \& Bögels, S. M. (2016). Mindful parenting training in child psychiatric settings: Heightened parental mindfulness reduces parents's and children's psychopathology. Mindfulness, 7(3), 680-689. doi: 10.1007/s12671-016-0504-1

Moreira, H., \& Canavarro, M. C. (2017). Psychometric properties of the Interpersonal Mindfulness in Parenting Scale in a sample of Portuguese mothers. Mindfulness, 8(3), 691-706. doi: 10.1007/ s12671-016-0647-0

Moreira, H., Carona, C., Silva, N., Nunes, J., \& Canavarro, M. C. (2015). Exploring the link between maternal attachment-related anxiety and avoidance and mindful parenting: The mediating role of selfcom- passion. Psychology and Psychotherapy: Theory, Research and Practice. doi:10.1111/papt.12082

Moreira, H., Fonseca, A., Caiado, B., \& Canavarro, M. C. (2019). Workfamily conflict and mindful parenting: the mediating role of parental psychopathology symptoms and parenting stress in a sample of Portuguese employed parents. Frontiers in Psychology, 10(635). doi: 10.3389/fpsyg.2019.00635

Morris, A. S., Silk, J. S., Steinberg, L., Myers, S. S., \& Robinson, L. R. (2007). The role of the family context in the development of emotion regulation. Social Development (Oxford, England), 16(2), 361-388.

Neff, K. (2003). The development and validation of a scale to measure self-compassion. Self and Identity, 2(3), 223-250. doi:10.1080/15298860309027
Nunes, L., \& Dewes, J. (2013) Amostragem em bola de neve e respondent-driven sampling: Uma descrição dos métodos. Trabalho de Conclusão de Curso de Graduação, Departamento de Estatística, UFRGS. Recuperado de http://hdl.handle. net $/ 10183 / 93246$

Parent, J., McKee, L., Rough, J., \& Forehand, R. (2016). The association of parent mindfulness with parenting and youth psychopathology across three developmental stages. Journal of Abnormal Child Psychology, 44(1), 191-202. doi:10.1007/ s10802-015-9978-x

Pasquali, L. (2003). Psicometria: Teoria dos testes na psicologia e na educação. Rio de Janeiro: Vozes.

Singh, N. N., Lancioni, G. E., Winton, A. S. W., Singh, J., Curtis, W. J., Wahler, R. G., \& McAleavey, K. M. (2007). Mindful parenting decreases aggression and increases social behavior in children with developmental disabilities. Behavior Modification, 31(6), 749-771.

Singh, N. N., Lancioni, G. E., Winton, A. S. W., Singh, J., Singh, A. N., Adkins, A. D., \& Wahler, R. G. (2010). Training in mindful caregiving transfers to parent-child interactions. Journal of Child and Family Studies, 19, 167-174.

Williams, K. L., \& Wahler, R. G. (2010) Are mindful parents more authoritative and less authoritarian? An analysis of clinic-referred mothers. Journal of Child and Family Studies. 19(2):230-235.

Recebido em: 18/12/2018

Reformulado em: 01/09/2020

Aprovado em: 14/09/2020 
Sobre os autores:

Lauren Frantz Veronez é Psicóloga (PUCRS), Especialista em Terapias Comportamentais Contextuais (CEFI/ CIPCO) e Mestre em Psicologia e Saúde (UFCSPA). Atua como psicóloga clínica e como professora convidada em cursos de especialização em todo o país.

ORCID: https://orcid.org/0000-0001-7397-7857

E-mail: laurenfv@gmail.com

Felipe Valentini é Doutor em Psicologia, é professor do Programa de Pós-Graduação Stricto Sensu em Psicologia da Universidade São Francisco (USF). Seus interesses de pesquisa focam em psicometria (principalmente TRI, SEM, análise multinível e viés de resposta), avaliação psicológica, inteligência e desempenho.

ORCID: https://orcid.org/0000-0002-0198-0958

E-mail: valentini.felipe@gmail.com

Caroline Tozzi Reppold é Doutora em Psicologia. Professora Associada da Universidade Federal de Ciências da Saúde de Porto Alegre. Coordenadora do Laboratório de Pesquisa em Avaliação Psicológica/UFCSPA. Bolsista Produtividade de Pesquisa do CNPq.

ORCID 0000-0002-0236-2553

E-mail: carolinereppold@yahoo.com.br

Janaína Thais Barbosa Pacheco é Doutora em Psicologia, Professora adjunta do Curso de Psicologia da Universidade Federal de Ciências da Saúde de Porto Alegre (UFCSPA). Professora e orientadora do Programa de Pós Graduação em Psicologia e Saúde da UFCSPA.

ORCID: http://orcid.org/0000-0001-5608-7457

E-mail: janaina.thais.pacheco@gmail.com

Contato com os autores:

Lauren Frantz Veronez

General Rondon, 1120/503

Porto Alegre-RS, Brasil

CEP: 91900-121 